\title{
Preparation and characterization of cobalt and copper oxide nanocrystals
}

\author{
A. H. MOHARRAM* \\ Physics Dept., College of Science \& Arts, King Abdulaziz Univ., Jeddah, Saudi Arabia
}

\begin{abstract}
Copper oxide and cobalt oxide $\left(\mathrm{Co}_{3} \mathrm{O}_{4}, \mathrm{CuO}\right)$ nanocrystals $(\mathrm{NCs})$ have been successfully prepared using microwave irradiation. The obtained powders of the nanocrystals (NCs) were characterized by X-ray diffraction (XRD), transmission electron microscopy (TEM), thermogravimetric (TGA) analysis and Fourier-transform infrared spectroscopy. The obtained results confirm the presence of both nanooxides which have been produced during chemical precipitation using microwave irradiation. TEM micrographs have shown that the obtained nanocrystals are characterized by high dispersion and narrow size distribution. The results of X-ray diffraction confirmed those obtained from the transmission electron microscope. Optical absorption analysis indicated the direct band gap for both kinds of the nanocrystals.
\end{abstract}

Keywords: nanoparticles synthesis; metal oxides; microwave oven; TEM micrographs; X-ray diffraction

\section{Introduction}

Nanocrystalline semiconductors have drawn considerable interest in recent years because of their special properties, such as a large surface to volume ratio and unique optical properties as compared to those of the bulk materials [1]. The colloidal NCs integrated in the epitaxial grown layers allow device implementation based on NCs [2]. Wet colloidal NCs prepared chemically exhibit an enormous potential regarding tuning of their optical and structural properties [3]. The oxides of transition metals represent an important class of semiconductors, which find applications in magnetic storage media, solar energy transformation, electronics and catalysis [4]. Copper oxide has attracted much attention because it is the basis of several high- $\mathrm{T}_{\mathrm{c}}$ superconductors. However, cobalt oxide is a promising candidate as an anode material for lithium secondary batteries because of its electrochemical capacity and high recharging rate [5]. The microwave synthesis, which is generally fast, simple and efficient in energy usage, has been developed and widely used in various fields, such as

*E-mail: mohar200@yahoo.com molecular sieve preparation, radiopharmaceuticals, preparation of inorganic complexes, organic reactions, plasma chemistry, analytical chemistry and catalysis [6]. In the present article, a rapid growth method has been used to prepare different monoclinic metal oxide nanocrystals using microwave irradiation. It was proved to be convenient, efficient and environmental friendly one step route to produce nanooxides. The product had good crystallinity, uniform morphology and high purity. The structural, optical and magnetic properties were studied for both kinds of nanoparticles.

\section{Experimental}

In a typical procedure, $25 \mathrm{~mL}$ water solution of $0.2 \mathrm{M} \mathrm{Cu}\left(\mathrm{NO}_{3}\right)_{2} \cdot 6 \mathrm{H}_{2} \mathrm{O}$ was added to $25 \mathrm{~mL}$ water solution containing $0.2 \mathrm{M} \mathrm{CO}\left(\mathrm{NH}_{2}\right)_{2}$ in a roundbottom flask. The obtained solution was thoroughly mixed, to reach molecular level homogenization of the reaction medium. The mixture was placed in a microwave oven with $650 \mathrm{~W}$ power and the reaction was carried out under ambient air for $10 \mathrm{~min}$. An excess of urea was used as fuel for the combustion and added under magnetic stirring for about $30 \mathrm{~min}$. As the ignition occurred, the reaction went 
on vigorously for a few seconds. Urea works as a basic medium to form copper hydroxide as shown by the following reaction:

$$
\begin{aligned}
& 3 \mathrm{Cu}\left(\mathrm{NO}_{3}\right)_{2} \cdot 6 \mathrm{H}_{2} \mathrm{O}+5 \mathrm{CO}\left(\mathrm{NH}_{2}\right)_{2}= \\
& =3 \mathrm{Cu}(\mathrm{OH})_{2}+8 \mathrm{~N}_{2}+25 \mathrm{H}_{2} \mathrm{O}+5 \mathrm{CO}_{2}, \\
& \mathrm{Cu}(\mathrm{OH})_{2}=\mathrm{CuO}(\mathrm{NCs})+\mathrm{H}_{2} \mathrm{O}
\end{aligned}
$$

A black fine powder of $\mathrm{CuO} \mathrm{NCs}$ was extracted. After cooling to room temperature, the precipitate was centrifuged, washed sequentially in distilled water, absolute ethanol and acetone and dried in air at room temperature. The final products were collected for characterization. The same steps were done for preparing the $\mathrm{Co}_{3} \mathrm{O}_{4} \mathrm{NCs}$ as follows:

$$
\begin{aligned}
& 3 \mathrm{Co}\left(\mathrm{NO}_{3}\right)_{2} \cdot 6 \mathrm{H}_{2} \mathrm{O}+5 \mathrm{CO}\left(\mathrm{NH}_{2}\right)_{2}= \\
& =3 \mathrm{Co}(\mathrm{OH})_{2}+8 \mathrm{~N}_{2}+25 \mathrm{H}_{2} \mathrm{O}+5 \mathrm{CO}_{2}, \\
& 3 \mathrm{Co}(\mathrm{OH})_{2}=\mathrm{Co}_{3} \mathrm{O}_{4}(\mathrm{NCs})+2 \mathrm{H}_{2} \mathrm{O}+\mathrm{H}_{2} .
\end{aligned}
$$

XRD measurements were performed on the powdered specimens using an X-ray (Shimadzu XD-3A) diffractometer. Copper target with $\lambda=1.5418 \AA$ was used as a source of X-ray. The scanning angle $2 \theta$ was in the range of $20^{\circ}$ to $70^{\circ}$. TEM micrographs were recorded using a transmission electron microscope (JEOL-200CX), with accelerating voltage of $80 \mathrm{kV}$. The energy dispersive spectrometry (EDS) analysis for detecting the characteristic X-rays of the constituent elements was carried out using an X-Max 80 detector unit which was equipped with the transmission electron microscope.

The thermal behavior was studied using Shimadzu differential scanning calorimetry. IR spectra were obtained using FT-IR (Nicolet NEXUS 670) spectrometer, which is characterized by high rapid-scan capability and equipped with the photoacoustic (Model 300 of MTEC) detector. The spectra of the oxide samples were recorded in nearIR region, $400 \mathrm{~cm}^{-1}$ to $4000 \mathrm{~cm}^{-1}$. The resolution of the measurements was $8 \mathrm{~cm}^{-1}$ and each spectrum was obtained by recording 200 consecutive scans. Optical properties of the nanoparticles in solution were studied using the UV-Vis (Shimadzu UV-2450) spectrophotometer in the wavelength range of $200 \mathrm{~nm}$ to $900 \mathrm{~nm}$.

\section{Results and discussion}

To understand the phase symmetry of the asprepared specimens, systematic XRD studies were performed. Fig. 1 shows the XRD patterns of the $\mathrm{CuO}$ and $\mathrm{Co}_{3} \mathrm{O}_{4}$ nanocrystals. The intensities and angular positions of the peaks are in good agreement with the previously mentioned values $[7,8]$. No peaks related to impurities have been found in the diffractograms. In Fig. 1a, the diffraction pattern is in agreement with JCPDS Card No. 5-661. The obtained lattice parameters $\mathrm{a}=4.653 \mathrm{~nm}, \mathrm{~b}=3.410 \mathrm{~nm}$ and $\mathrm{c}=5.108 \mathrm{~nm}$ indicate the monoclinic structure of the $\mathrm{CuO}$ nanocrystals [9] which was found to be highly crystalline. The sharp X-ray peaks of the second $\mathrm{Co}_{3} \mathrm{O}_{4}$ product (Fig. 1b) indicate the monoclinic structure of the nanocrystals which was also found to be highly crystalline in agreement with the JCPDS Card No. 78-1969. Stability of the nanoparticles products can be easily checked using even one method, such as X-ray diffraction. When the sharpness degree of the diffraction peaks does not change over long time it confirms the stability of the present products. The average size of the nanocrystals can be estimated using the Debye-Scherrer formula [10]:

$$
D=k \lambda / \beta \cos \theta
$$

where $\mathrm{k}$ is a constant equal to $0.94, \lambda$ is the wavelength of the $\mathrm{X}$-ray radiation $(\lambda=1.5418 \AA)$, and $\beta$ is the full width at half maximum of the diffraction peak corresponding to $2 \theta$. Using equation 3 , the crystallite sizes were found to be in the range of $14 \pm 1 \mathrm{~nm}$ and $14.5 \pm 1.3$ for $\mathrm{CuO}$ and $\mathrm{Co}_{3} \mathrm{O}_{4}$ nanocrystals, respectively.

Fig. 2 shows the TEM images of the asprepared $\mathrm{CuO}$ and $\mathrm{Co}_{3} \mathrm{O}_{4}$ nanocrystals. TEM micrographs have revealed that both oxides are composed of spherical particles with regular morphology and narrow size distribution. These findings are in agreement with those reported in previous studies $[11,12]$. The particle size observed in TEM micrographs is $10 \pm 2 \mathrm{~nm}$ and $13 \pm 2$ for $\mathrm{CuO}$ and $\mathrm{Co}_{3} \mathrm{O}_{4}$ nanocrystals, respectively, which is in good agreement with the results calculated by the Debye-Scherrer formula. The strain induced 


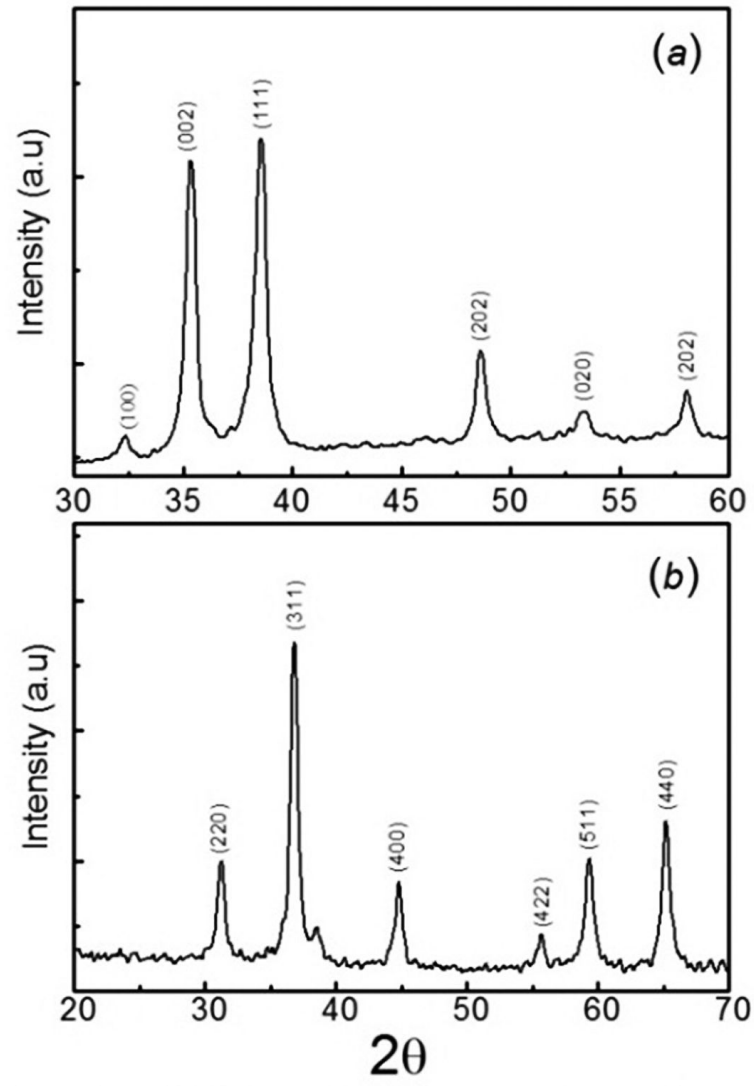

Fig. 1. XRD patterns of the as-prepared (a) $\mathrm{CuO}$ and (b) $\mathrm{Co}_{3} \mathrm{O}_{4}$ nanocrystals.

in powders due to the crystal imperfection and distortion [13] can be calculated using the following formula:

$$
\varepsilon=\beta / 4 \tan \theta
$$

The average values of induced strain are 0.0028 and 0.0027 for $\mathrm{CuO}$ and $\mathrm{Co}_{3} \mathrm{O}_{4}$ nanocrystals, respectively. These strain values have been assumed to be uniform in all crystallographic directions, considering the isotropic nature of the crystal, where the material properties are independent of the direction along which they are measured.

Fig. 3 shows the EDX analysis of CuO-NPs annealed at $600{ }^{\circ} \mathrm{C}$. The results reveal the presence of copper $(\mathrm{Cu})$ and oxygen $(\mathrm{O})$ elements in the products and indicate that the nanoparticles are nearly stoichiometric. There are no other elemental impurities in the EDX spectra. The EDX result shows the presence of uniform distribution of copper

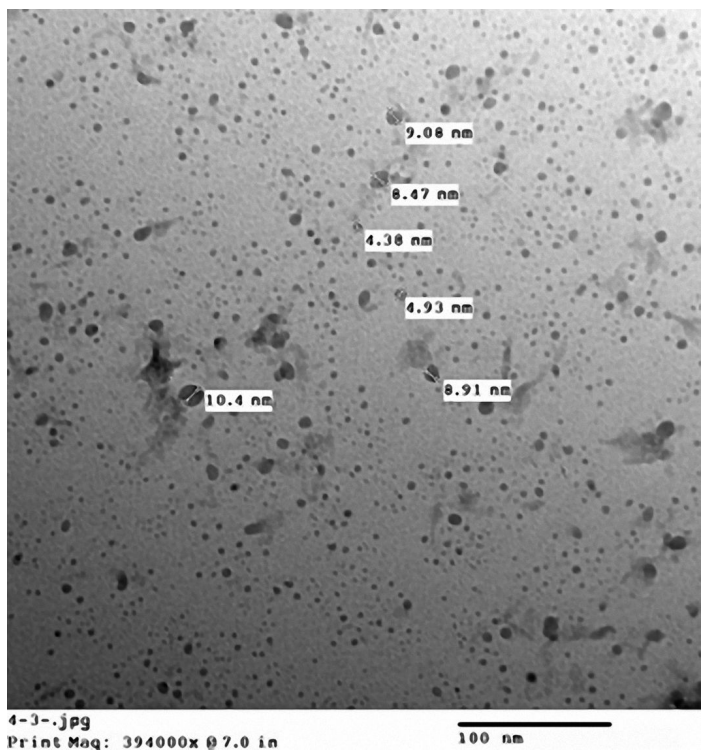

(a)

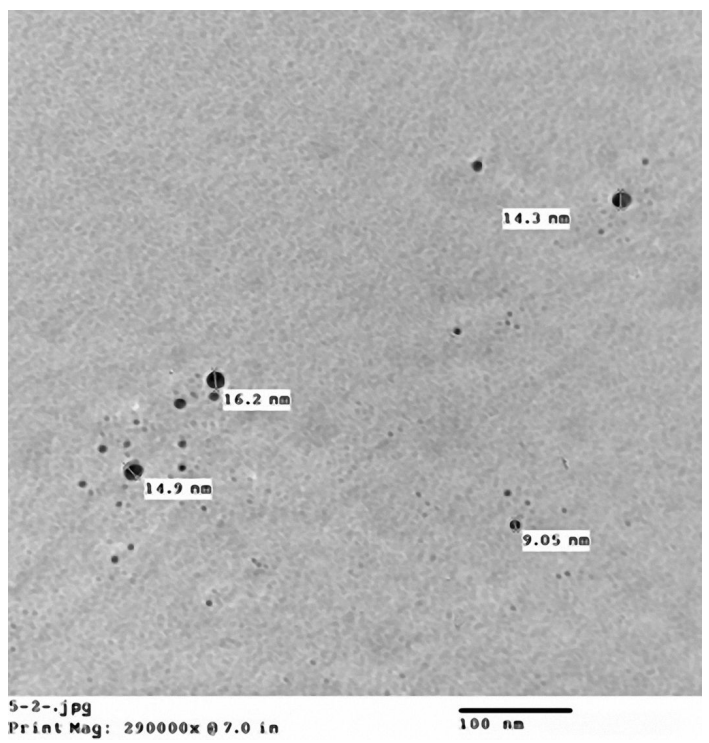

(b)

Fig. 2. TEM micrographs of the as-prepared (a) $\mathrm{CuO}$ and (b) $\mathrm{Co}_{3} \mathrm{O}_{4}$ nanocrystals.

and oxygen with atomic ratio of $1: 1 \mathrm{in} \mathrm{CuO}$. This result confirms the formation of pure CuO-NPs. The elemental analysis of the sample shows that the prepared sample is copper oxide, which is in good agreement with the results of XRD. Similar results were reported in the literature [14].

Temperature dependent weight loss of the asprepared $\mathrm{CuO}$ and $\mathrm{Co}_{3} \mathrm{O}_{4}$ nanocrystals have been 


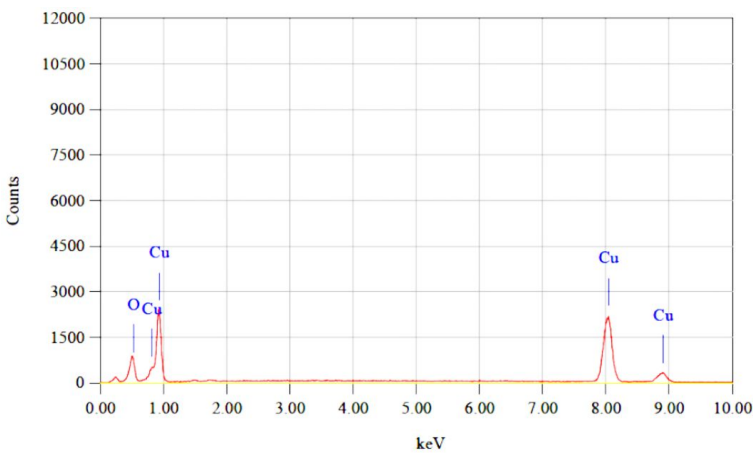

Fig. 3. Energy dispersive X-ray analysis of CuO-NPs annealed at $600^{\circ} \mathrm{C}$.

analyzed and the obtained results are shown in Fig. 4. It is easily seen from the TGA curves that the mass loss process of both oxides starts at $75^{\circ} \mathrm{C}$, which is related to evaporation of the water molecules absorbed on the surface of the products. In the same figure, another mass loss appears at about $205{ }^{\circ} \mathrm{C}$ which is due to decomposition of the $\mathrm{Cu}(\mathrm{OH})_{2}$ molecules.

Fourier transform infrared (FT-IR) spectrometry has been applied to study the surfaces of the $\mathrm{CuO}$ and $\mathrm{Co}_{3} \mathrm{O}_{4} \mathrm{NCs}$ in the wavenumber range $400 \mathrm{~cm}^{-1}$ to $4000 \mathrm{~cm}^{-1}$. In case of the $\mathrm{CuO}$ products, three distinctive bands have appeared as depicted in Fig. 5a.

The first strong absorption band at $533 \mathrm{~cm}^{-1}$ is due to vibrations of $\mathrm{CuO}$ functional groups, which confirms the presence of nanosized $\mathrm{CuO}$ particles in the nitrate matrix. The second band at $1104 \mathrm{~cm}^{-1}$ is related to the occurrence of $\mathrm{O}-\mathrm{C}-\mathrm{O}$ tensional tremble. Finally, the third one, corresponding to $\mathrm{C}=\mathrm{O}$ stretching of carboxylate ion bond to the $\mathrm{CuO}$ nanoparticles as bidentate ligand, is located at $1383 \mathrm{~cm}^{-1}$. Coordination of bidentate type is expected between the carboxylate ion and the $\mathrm{Cu}$ (II) of $\mathrm{CuO}$ [15]. As shown in Fig. 5b, it displays three distinctive bands originating from the stretching vibrations of metal-oxygen bond. The first band at $568 \mathrm{~cm}^{-1}$ is associated with the BOB3 vibrations in the spinel lattice, where $\mathrm{B}$ denotes the Co cations in an octahedral position, i.e. $\mathrm{Co}^{3+}$ ions. The second band at $662 \mathrm{~cm}^{-1}$ is attributed to the $\mathrm{ABO} 3$ vibrations, where $\mathrm{A}$ denotes the metal ion in a tetrahedral position. The third

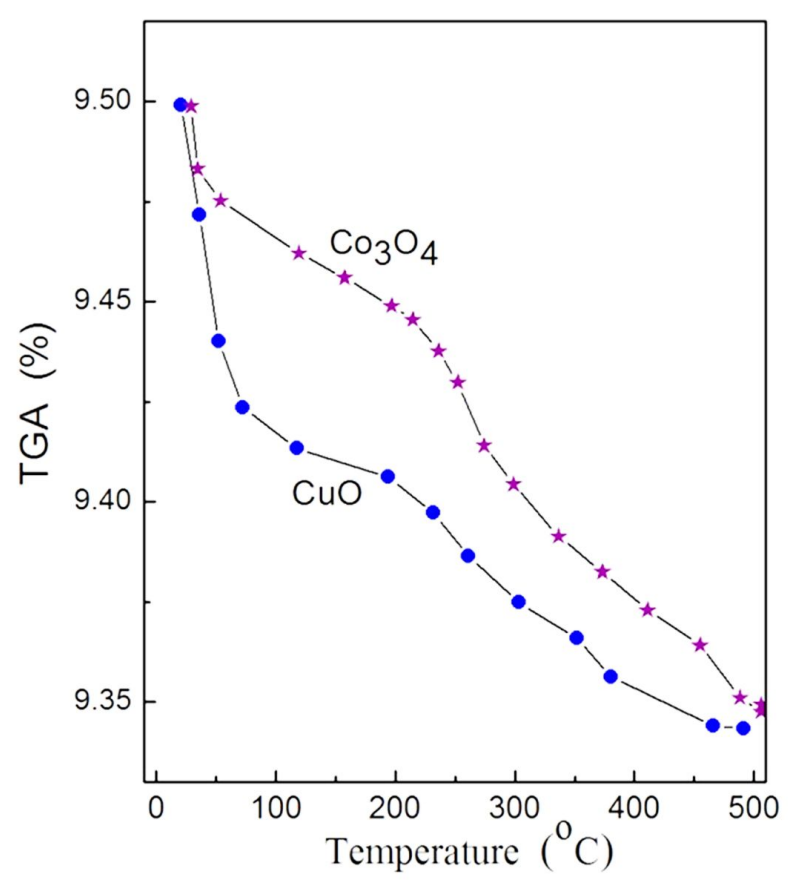

Fig. 4. TGA analysis curve for as-prepared (a) $\mathrm{CuO}$ and (b) $\mathrm{Co}_{3} \mathrm{O}_{4}$.

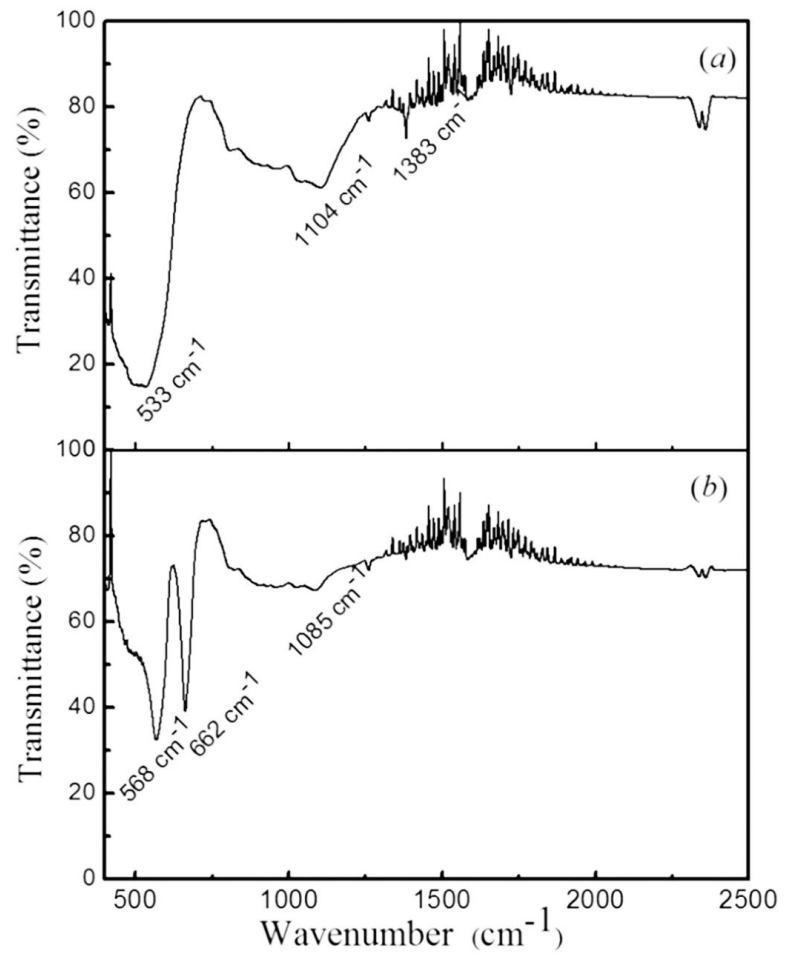

Fig. 5. FT-IR spectra of the as-prepared (a) $\mathrm{CuO}$ and (b) $\mathrm{Co}_{3} \mathrm{O}_{4}$ nanocrystals in the range of $400 \mathrm{~cm}^{-1}$ to $2500 \mathrm{~cm}^{-1}$. 
band at $1085 \mathrm{~cm}^{-1}$ is related to the occurrence of $\mathrm{O}-\mathrm{C}-\mathrm{O}$ tensional tremble.

UV-Vis electronic absorption spectroscopy study of the as-prepared oxides has been performed. The optical absorption spectra [16] have been used to calculate the absorption band gap $\mathrm{E}_{\mathrm{o}}$ according to the following equation:

$$
(\alpha h v)^{n}=B\left(h v-E_{o}\right)
$$

where $\mathrm{h} v$ is photon energy, $\alpha$ is the absorption coefficient, $\mathrm{B}$ is a constant related to the material and the exponent $\mathrm{n}$ is equal to either 2 for direct transition or $1 / 2$ for an indirect transition. Fig. 6 shows the $(\alpha \mathrm{h} v)^{2}$ versus $\mathrm{h} v$ plots for both $\mathrm{CuO}$ and $\mathrm{Co}_{3} \mathrm{O}_{4}$ nanocrystals.

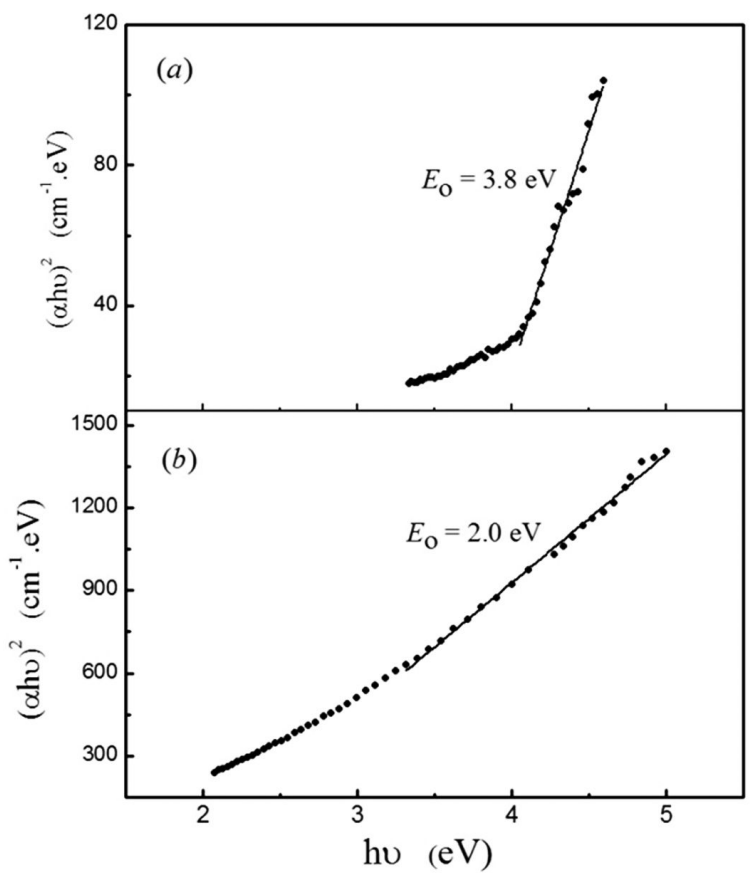

Fig. 6. $(\alpha \mathrm{h} v)^{2}$ as a function of photon energy for the asprepared (a) $\mathrm{CuO}$ and (b) $\mathrm{Co}_{3} \mathrm{O}_{4}$ nanocrystals.

The band gap of the as-prepared $\mathrm{CuO}$ was estimated to be $2.3 \mathrm{eV}$ and $3.8 \mathrm{eV}$. The former value $(2.3 \mathrm{eV})$ is higher than that measured $\left(\mathrm{E}_{\mathrm{o}}=1.85 \mathrm{eV}\right)$ for bulk $\mathrm{CuO}$ [17] but lower than that reported for the $\mathrm{CuO}$ nanocrystals [18]. Extrapolation of the straight segment of the obtained curve in Fig. $5 \mathrm{~b}$, gives $\mathrm{E}_{\mathrm{o}}=2.0 \mathrm{eV}$ for direct allowed transition of $\mathrm{Co}_{3} \mathrm{O}_{4}$ nanocrystals which is little larger than that reported for bulk $\mathrm{Co}_{3} \mathrm{O}_{4}\left(\mathrm{E}_{\mathrm{o}}=1.96 \mathrm{eV}\right)$ specimens [19]. Similar results of the optical bang gaps for both $\mathrm{CuO}$ and $\mathrm{Co}_{3} \mathrm{O}_{4}$ nanocrystals have been reported in the literature [20]. These band gap values are higher than the values of the bulk samples which is due to the quantum confinement effect.

\section{Conclusions}

Nanocrystalline $\mathrm{CuO}$ and $\mathrm{Co}_{3} \mathrm{O}_{4}$ particles with a monoclinic structure have been prepared successfully by a novel method using microwave irradiation. From XRD and TEM studies, it has been found that the particles are spherical in shape with an average size of $14 \mathrm{~nm}$. Analyses of the FT-IR data have revealed the formation of impure $\mathrm{CuO}$ and $\mathrm{Co}_{3} \mathrm{O}_{4}$ phases with monoclinic crystal structure. The optical properties of both nanocrystals revealed the direct band gap of $2.3 \mathrm{eV}$ for the copper oxide particles and $2.0 \mathrm{eV}$ for the cobalt oxide product. These values of the band gap are higher than the values of the bulk samples which is due to the quantum confinement effect.

\section{Acknowledgements}

This research was funded by the Deanship of Scientific Research (DSR) at King Abdulaziz University, Jeddah, under Grant No. 662-546-D1435. The author, therefore, acknowledges with thanks DSR for technical and financial support.

\section{References}

[1] Henglein A., Chem. Rev., 89 (1989), 1861.

[2] KAMPMEIER J., RASHAD M., WOggon U., Ruth M., Meier C., Schikora D., LischKa K., Pawlis A., Phys. Rev. B, 85 (2012), 155405.

[3] Rashad M., Paluga M., Pawlis A., LischKa K., Schikora D., Artemyev M. V., Woggon U., Phys. Stat. Sol. C, (2010), 1.

[4] IJaz F., Shahid S., Khan S.A., Ahmad W., ZaMAN S., Tropical J. Pharmac. Res., 16 (2017), 743.

[5] Poizot P., Laruelle S., Grugeon S., Dupont L., Nature, 407 (2000), 496.

[6] Saskia A.G., Chem. Soc. Rev., 269 (1997), 233.

[7] JadhaV S., GaikWAd S., Nimse M., RajBhoJ A., $J$. Clust. Sci., 22 (2011), 121.

[8] Sankar R., Manikandan P., Malarvizhi V., FATHIMA T., Shivashangari K.S., RaViKumar V., Spectrochim. Acta Mol. Biomol. Spectrosc., 121 (2014) 746. 
[9] Yao W.T., Yu S.H., Zhou Y., Jiang J., Wu Q.S., Zhang L., Jiang J., J. Phys. Chem. B, 109 (2005), 14016.

[10] Klug H., Alexander L., X-ray Diffraction Procedures, Wiley, New York, 1962, p. 125.

[11] Das SK, Khan MMR, GuHAB AK, Naskar N., Green Chem., 15 (2013), 2548.

[12] Saif S., Tahir A., Asim T., Chen Y., Nanoma., 6 (2016), 1.

[13] Mote V.D., Purushotham Y., Dole B.N., J. Theor. Appl. Phys., 6 (2012).

[14] Manimaran R., Palaniradja K., Alagumurthi N., Sendhilnathan S., Hussain J., Appl. Nanosci., 4 (2014), 163.
[15] SOCRATES G., Group Frequencies - Tables and Charts, $2^{\text {nd }}$ ed., John Wiley and Sons, England, 1994.

[16] Pankove J.I., Optical processes in semiconductors. Englewood Cliffs, Prentice-Hall, New York, 1971.

[17] Santra K., Sarkar C.K., MukherJee M. K., Cosh B., Thin Solid Films, (1992) 213.

[18] Zhu J.W., Chen H.Q., LiU H.B., YANG X.J., LU L.D., WANG X., Mater Sci. Eng. A, 384 (2004), 172.

[19] Drasovean R., J. Non-Cryst. Solids, 352 (2006), 1479.

[20] Gu F., KI C., Hu Y., Zhang L., J. Cryst. Growth, 304 (2007), 369.

Received 2017-06-14 Accepted 2018-11-12 